\title{
A Roadmap to Retention Protocols Applicable to Various Clinical Cases: An Overview
}

\author{
Dweepika Garg, Santosh Kumar Goje \\ Department of Orthodontics and Dentofacial Orthopaedics, K. M. Shah Dental College and Hospital, Sumandeep Vidyapeeth, \\ Vadodara - 390 011, Gujarat, India
}

Email for correspondence: dweepika_dg1@yahoo.co.in

\begin{abstract}
In orthodontics, the stability of the achieved result remains primary concern and debate. Usually, a retention phase is required after active orthodontic tooth movement to hold teeth in their esthetic and functional relation and to prevent them in returning to their former position. Therefore, it is of utmost importance to retain the teeth by appropriate appliance. Since each case is different, it requires a unique retentive protocol and appliance. The present article enlists various retention protocols among various clinical situations including orthognathic surgeries.
\end{abstract}

Key words: Clear vacuum-formed retainers, Hawley retainers, retention, retentive appliances, wrap around clip retainers

\section{INTRODUCTION}

Retention is the phase in orthodontic treatment that aims in maintaining the teeth in their orthodontically corrected positions following cessation of active orthodontic tooth movement. Orthodontic retainers act by resisting the tendency of the teeth to return to their original pre-treatment positions under the influence of occlusal and soft tissue forces and periodontal and continuing dentofacial growth. ${ }^{[1]}$

Since each case is unique, it requires a unique retention plan, one plan does not fit in all the situations. Therefore, it is important to analyze which retainer will suit which situation. Choice of retainer is not only governed by the clinical situation but by patients compliance also; patient's compliance is another major factor which determines the choice of retainer.

Retention should be considered right from the beginning of diagnosis and treatment planning. Most of the clinicians would agree to this. In doing so, the

\begin{tabular}{|l|l|}
\hline Quick Response Code & Article Info: \\
\hline doi: 10.5866/2018.10.10035 \\
Received: $28-12-2017$ \\
Revised: $27-01-2018$ \\
Accepted: 08-02-2018 \\
Available Online: $15-04-2018,2018$ (www. \\
nacd.in)@ NAD, 2017 - All rights reserved
\end{tabular}

potential factors considered for long-term stability will be kept in mind throughout the treatment. Setting the retentive goals early is as important as the orthodontic biomechanical objectives of tooth movement in the craniofacial environment. ${ }^{[2]}$

Stability can be achieved only if the forces derived from the periodontal and gingival tissues, the orofacial soft tissues, the occlusal forces, and post-treatment facial growth and development are in equilibrium. ${ }^{[3]}$ The recommended length of time for wearing a retainer varies from orthodontist to orthodontist. The etiology of alignment instability following orthodontic treatment is still puzzling and influenced largely by individual factors and different retention protocols. ${ }^{[4]}$

Retainers can either be fixed to the teeth or removable. A fixed retainer consists of a thin stainless steel wire (approximately $1 \mathrm{~mm}$ diameter) that is bonded on the lingual surfaces of the incisor teeth with tooth colored adhesive. There are many types of removable retainers. ${ }^{[5]}$ These include acrylic Hawley retainer, clear vacuum-formed retainer (VFR), wrap around clip retainer, and positioners. ${ }^{[6]}$

\section{DIFFERENT SITUATIONS AND THEIR RETENTIVE PROTOCOL}

\section{Lower Incisor Crowding [Figure 1]}

The skeletal pattern must be considered during the formulation of a treatment plan. The patient 
with hyperdivergent skeletal pattern must have mandibular incisors that overly upright over basal bone to achieve a balanced face at the end of treatment and to ensure more stability of tooth alignment as the patient matures. If the incisors are not overly upright, the patient may have poor facial esthetics, compromising the stability of the dentition. On the other hand, in a patient with hypodivergent skeletal pattern, mandibular incisors may be left in their pre-treatment position. They should not be flared to correct a curve of Spee or to relieve crowding. Leaving them in their pre-treatment position protects the balance and harmony of the face ensuring the long-term stability of the dentition. ${ }^{[7]}$ Permanent retention to ensure stability of post-treatment outcome, especially for maintenance of mandibular incisor alignment, has become a common trend today. ${ }^{[8]}$

Rourke et al. carried out a prospective randomized controlled clinical trial to compare the clinical effectiveness of bonded retainers with vacuum-formed retainers, in terms of maintaining the results of orthodontic treatment in the lower arch up to 18 months' post debond. They investigated the effectiveness of these retainers in maintaining the stability of lower anterior teeth. They concluded that bonded retainers have a superior ability to hold the mandibular incisor alignment in the first 6 months after treatment as compared to the vacuum-formed retainers. $^{[9]}$

\section{Anterior Dental Crossbite [Figure 2]}

When the incisor overbite and posterior intercuspation are adequate for maintaining the correction, no retention protocol is required.$^{[1]}$ Early correction of anterior dental crossbite in the early mixed dentition is very important. Simple anterior dental crossbite can be easily treated either with a simple removable appliance or by a 24 (2 molars and 4 incisors) bonding technique, and almost in all cases, this crossbite correction needs no retention. Correction in itself serves as the retainer. However, a removable retainer can be given to the patient if deemed essential. ${ }^{[8]}$

\section{Deep Bite [Figure 3]}

Correcting a deep bite and stability of the result are a problem in orthodontic treatment. All deep bites are not alike and must be differentiated for proper treatment planning. Depending on etiological factors and the dentoskeletal structures involved in the malocclusion such as over eruption of incisors, molar

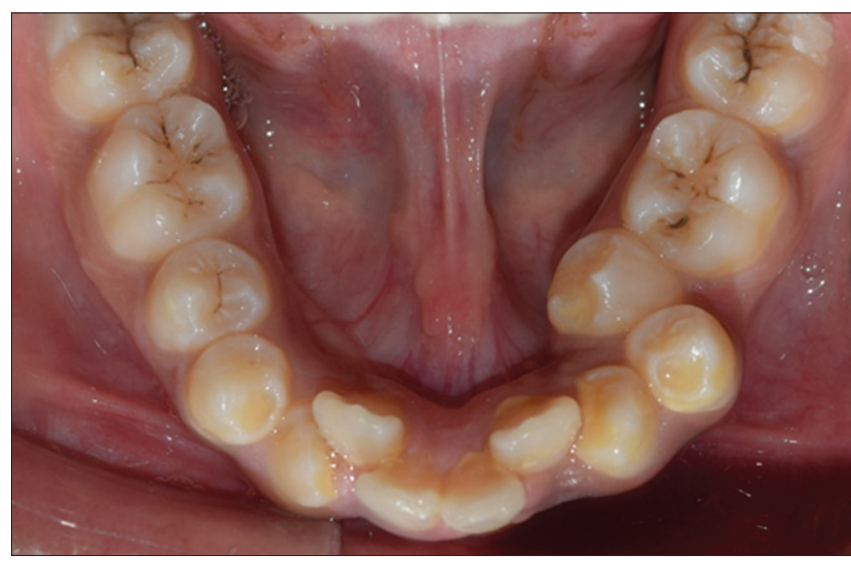

Figure 1: Clinical picture showing lower anterior crowding

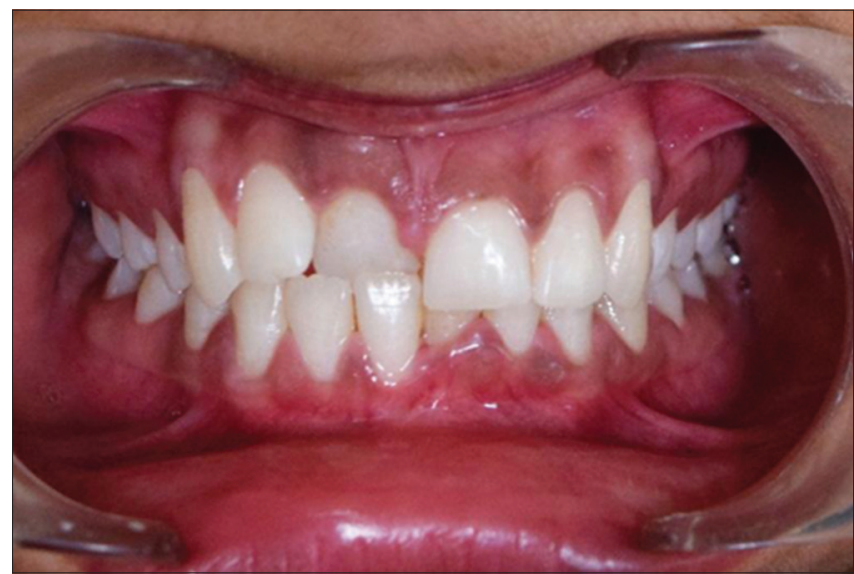

Figure 2: Clinical picture showing single tooth crossbite

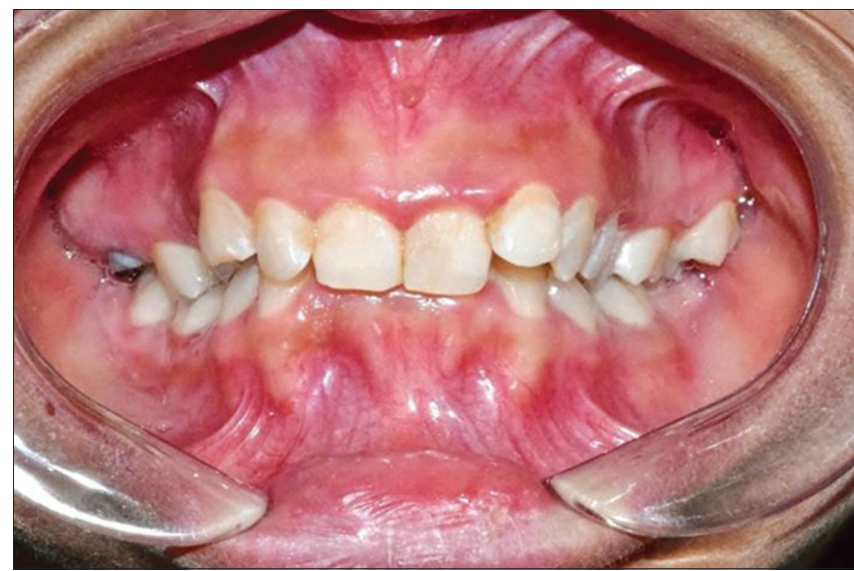

Figure 3: Clinical picture showing deep bite

intrusion or a combination, counterclockwise rotation of mandible, and maxillary clockwise rotation; various types of orthodontic mechanics are available for the overbite reduction. Early intervention is the key and such results are more stable. Simple is often the best 
and examples include the Hawley with anterior bite plane or fixed lingual retainers. ${ }^{[8]}$

Following the correction of a severe deep overbite, it is advised to use a passive anterior bite plane until the completion of facial growth. This may be particularly useful when there is evidence of an anterior mandibular growth rotation. ${ }^{[1]}$

\section{Open Bite}

Early intervention is easier and more stable, and depending on the structure targeted by our treatment, many types of preventive devices are usable such as lower or lingual holding arch and transpalatal arch, and a Hawley bite block can be used as retainer. These, all aim at restricting molar eruption and thus increasing the anterior overbite and usually also reduce the anterior facial height. ${ }^{[8]}$

\section{Rotated Anterior Teeth}

Supracrestal gingival fibers take the longest amount of time to reorganize. Therefore, prolonged retention of corrected rotations is required in reducing relapse. Circumferential supracrestal fiberotomy can be done either in the conventional way with the help of a surgical scalpel or with use of a laser-aided probe. It is used to transect the gingival fibers. Laser-aided probe is known to have various advantages such as reduced bleeding, minimal swelling, and no apparent damage to the supporting periodontal structures. ${ }^{[1]}$

\section{Spaced Dentition [Figure 4]}

After treating a case of generalized spacing or a midline diastema, permanent retention is recommended..$^{[1]}$

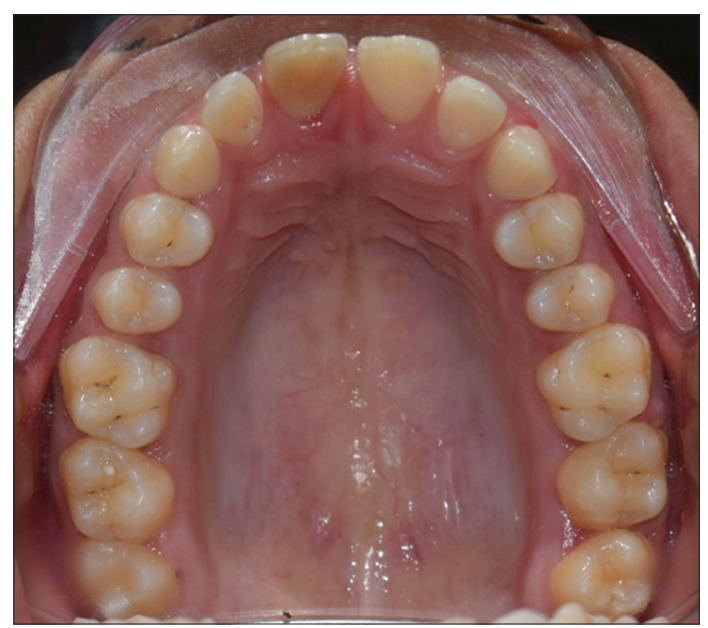

Figure 4: Clinical picture showing generalized spacing in the dentition
Nevertheless, this technique may lead to longterm harm if the appliance is left without any supervision. Unwanted side effects due to distorted fixed retainers are common and range from minor rotations to bone fenestrations and excessive displacement of the anchoring teeth. Furthermore, it is a known fact that oral hygiene gets compromised with fixed retainers. ${ }^{[4]}$

Cifter et al. evaluated the effects of VFRs on periodontal tissues and the retention efficiency of VFRs. They concluded that VFR led to clinical attachment loss over a prolonged period of time with the data being not significant clinically. In terms of stability, VFRs were found to be effective in orthodontic retention. ${ }^{[10]}$

\section{Adult Patients}

When the periodontal supporting tissues are normal and occlusal settling is not required, there is no evidence to support that any change in retention protocol is required for adult patients compared with adolescent patients. ${ }^{[1]}$

Despite the disadvantages of VFR, such as reduced vertical settling and occlusal wear, they are becoming extremely popular due to their improved esthetics, ease of application, reduced cost, and ease of fabrication. Adults prefer VFR due to added advantage of esthetics. ${ }^{[10]}$

Wan et al. carried out a study in adults where they assessed the effects of alteration on speech articulation between Hawley retainers and vacuumformed retainers by an objective acoustic analysis of vowels and voiceless fricatives. Acoustic analysis before and after retainer application was done to assess the speech sounds. They concluded that although sound distortion could be found in both the Hawley retainer group and the vacuum-formed retainer group, changes in articulation were more obvious in the Hawley retainer group. ${ }^{[11]}$

\section{ADVANTAGES AND DISADVANTAGES OF EACH RETAINER}

Hawley's retainer allows the masticatory force to getdistributedontoothsurface, therebyallowingminor teeth settling after debonding. The disadvantage of Hawley'sretaineristhatitisnotsuitableinpatientswith poor compliance. Being removable, it has to becleaned bythepatientproperlyandbreakageshouldbeavoided.

VFR has a major advantage of esthetics, ease of fabrication, and reduced cost but can only be given in cases which have excellent finish at debonding. Cases 
thatdonotrequireverticalsettlingafterdebondareideal forVFR sinceVFRdonotallow themuscularforcetoact onteeth,therebymaintainingtheobtainedrelationship. Themajor disadvantages ofVFRareocclusalwearand poor occlusal settling.

Fixedbondedretainerhasnoproblemwithpatient's compliance since it cannot be removed by the patient. Furthermore, thereisnocosmeticissuesinceitishidden behind theteeth. However, theonlydisadvantageis the difficultyincleaningandflossing.Poororalhygienecan lead to accumulation of plaque and calculus.

Therefore, aftergoingthroughall the prosandcons andafteranalyzingtheliterature,itcanbeconcludedthat no one retainer is ideal.

One technique which can be implemented in most ofthesituationsistogiveaHawleyretainertothepatient immediately after debonding. This will allow vertical settling of the occlusion as all the muscular force and occlusalforcecanactontheteeth.Afterverticalsettling isestablishedafter3-4 months, Hawleyretainercanbe changed toVFR. This will helpin retaining the teethin ideal position.

\section{RETENTIONAFTERSURGICALORTHODONTICS}

Retention regimens have become an integral part of the present-day orthodontic treatment plan. The ultimate success of the long-term orthodontic treatment result depends on a compilation of steps, including appropriate planning, well-controlled treatment mechanics, retention compliance, and an appreciation of the biological limits of tooth movement. ${ }^{[12]}$

Not all cases can be corrected by orthodontic treatment alone. In severe skeletal malformations, a surgical approach would be required. Thus, orthognathic surgery is undertaken. Conventionally, maxillofacial deformities are corrected surgically after an initial orthodontic treatment phase. ${ }^{[13]}$

An interdisciplinary approach between the orthodontist and maxillofacial surgeon is imperative to successfully plan and execute a comprehensive treatment plan with predictable outcomes. ${ }^{[14]}$

Farronato et al. emphasized the post-surgical therapeutic protocol which determines the final and permanent retention of the corrected occlusion. ${ }^{[13]}$ Combined surgical and orthodontic correction of the malocclusion was used. Restoration and rehabilitation of neuromuscular function, grinding teeth selectively, obtaining occlusal stabilization, and final occlusion retention were the goals of post-operative therapy.
The importance of a surgical occlusal splint for rehabilitating stomatognathic neuromuscular function postoperatively was shown. The long-term results confirmed the efficacy of the treatment protocol from both functional and esthetical perspectives.

The correct control of the postsurgical orthodontic phase is as important as the presurgical orthodontic phase in surgical-orthodontic treatment. The initial diagnosis, exact planning, and execution of the orthognathic surgery determine the result. To perfect the dental occlusion relative to the new skeletal relationships, post-operative orthodontic therapy is used. It is important to restore neuromuscular function through reprogramming of muscular and dental-periodontal proprioception which is adequate for the new spatial situation of the maxillary and mandibular skeletal bases. Esthetics, function, stability, and treatment time are key factors to be considered for the decisionmaking process. Orthognathic surgery is important when it is considered as part of the therapeutic method. ${ }^{[13]}$

Orthognathic surgery to reposition the maxilla, mandible, or chin is the chief treatment for patients who are too old for growth modification and for dentofacial conditions that are too severe for either surgical or orthodontic camouflage. ${ }^{[14]}$

Surgical procedures are also carried out to correct cases of skeletal class III. Akan et al. published a case of 25-year-old female patient who underwent BSSO. ${ }^{[15]}$ The results were stable even after 8 years. Rigid bicortical screw fixation was done. Furthermore, being 25 years old, there was no late mandibular growth left in the patient. Thus, they concluded that mandibular set back surgery should be avoided at younger age. Profit has compared postsurgical stability after mandibular set back using three techniques. He concluded that rigid fixation after surgery is necessary or BSSO with wire synthesis can also be done. ${ }^{[16]}$

With the advent of rigid internal fixation across the osteotomy site, uncontrolled skeletal relapse is unlikely to occur. Skeletal remodeling at the site of osteotomy and the mandibular condylar heads may continue up to 6-12 months' postoperatively. ${ }^{[17]}$

There are few orthognathic surgeries which are extremely stable and few others which are prone to relapse. Profit compared the different surgical procedures and concluded the most and 
least stable procedure. ${ }^{[16]}$ The hierarchy of stability was analyzed, and it was concluded that superior repositioning of maxilla was the most stable procedure, with transverse expansion of maxilla being the least stable. The combination of moving the maxilla upward and forward movement of the mandible is significantly more stable when rigid internal fixation is used in the mandible.

In an updated article, hierarchy of stability with rigid fixation was analyzed by profit. ${ }^{[18]}$ Two procedures which were not included earlier were now placed: correction of asymmetry is stable with rigid fixation and repositioning of the chin also is very stable. Surgical movements for Class II with long face problems tend to be more stable than those treated for Class III problems. Since the dentition adapts to the skeletal change, long-term dental changes were fewer.

Orthognathicsurgery relies on aclosecollaboration between the surgeon and the orthodontist across all stages of treatment, from pre-operative planning to finalization of occlusion. ${ }^{[14]}$

\section{DISCUSSION}

Radunovic et al., Norwegian orthodontist, conducted a survey wherein they surveyed the retention protocols and needed for practical retention guidelines. They concluded that the most common maxillary retainer was a combination of a fixed and removable retainer, followed by a clear thermoplastic retainer. In the mandible, a fixed retainer bonded to all anterior teeth was the most common. ${ }^{[19]}$

Another study by Pratt et al. was to evaluate retention protocols among members of the American Association of Orthodontists in the United States. A 36-question electronic survey on certain questions was used. The survey was sent to all practicing members of the American Association of Orthodontists in the US, and $18 \%$ responded. Mean retention protocols showed the use of Hawley or vacuum-formed retainers in the maxillary arch and fixed retention in the mandibular arch. There is a current shift away from Hawley retainers and toward vacuum-formed retainers and fixed retention for both the arches. ${ }^{[20]}$

According to a survey conducted in Netherlands by Anne Renkema, it was concluded that most orthodontists placed a bonded retainer in the upper and lower arch, except when the upper arch was expanded during treatment or when extractions were performed in the upper arch, in which case they placed a removable retainer. ${ }^{[21]}$ Opinions varied with regard to how many hours the removable retainers should be worn and the duration of the retention phase. Contraindications for bonded retainers were given by $96 \%$ of the orthodontists, with poor oral hygiene being the most commonly mentioned. As far as bonded retainers were concerned, $84 \%$ of the orthodontists preferred permanent retention. ${ }^{[12]}$

A systematic review was published by Mai et al. where they compared vacuum-formed and Hawley retainer. ${ }^{[22]}$ After searching five electronic databases without language restrictions from 1960 to July 2013, they concluded that there was no significant difference between vacuum-formed and Hawley retainers in retaining dentition in terms of intercanine and intermolar widths. No significant differences in arch lengths were reported. In one trial, vacuum-formed retainers were more effective than Hawley retainers for holding corrections of the maxillary and mandibular labial segments (Little's index of irregularity). There were higher rates of breakage with Hawley retainers.

One must ensure that the retainer is not only fulfilling its role of maintaining stability but also not causing any harm. It is the clinician's responsibility to advise the patient on the importance of this longterm maintenance and how best to do this. It is the patient's responsibility to follow this advice. ${ }^{[23]}$

\section{CONCLUSION}

Retention being the most important step in orthodontics, requires special consideration. The same retention plan does not fit all the cases. It should be planned for each patient from the beginning of diagnosis and treatment planning.

\section{REFERENCES}

1. Johnston C, Burden D, Morris D. Clinical guidelines: Orthodontic retention. Semin Orthod 2008;12(2):109-122.

2. Rossouw P, Malik S. The retention protocol. Semin Orthod 2017;23:237-48

3. Reddy R, Suma S, Chaukse A. Retention appliances-a review. Int J Dent Clin 2010;2:31-6.

4. Rody W, Wheeler T. Retention management decisions: A review of current evidence and emerging trends. Semin Orthod 2017;23:221-8

5. Lustig J, Rossouw P, Buschang P, Behrents R, Woody R. Assessment of post orthodontic occlusal contacts with wrap-around and clear overlay retainers. Semin Orthod. 2016;23:166-77.

6. Gill D. Orthodontic retention: What? Why? How? When? Semin Orthod 2008;14:173-26. 
7. Vaden J. Long term stability-it begins with the treatment plan. Semin Orthod 2017;23:149-65.

8. Bahreman A. Retention considerations in the assessment of long-term stability in early versus late orthodontic treatment. Semin Orthod 2017;23:123-48.

9. Rourke N, Albeedh H, Sharma P, Jonal A. Effectiveness of bonded and vacuum-formed retainers: A prospective randomized controlled clinical trial. Am J Orthod Dentofacial Orthop 2016; 150:406-15.

10. Cifter M, Celikel A, Cekici A. Effects of vacuum-formed retainers on periodontal status and their retention efficiency. Am J Orthod Dentofacial Orthop 2017;152:830-5.

11. Wan J, Wang T, Pei X, Wan Q, Feng W, Chen J. Speech effects of Hawley and vacuumformed retainers by acoustic analysis: A single-center randomized controlled trial. Angle Orthod 2017;87:286-92.

12. Rossouw P. The need for retention-an important consideration. Semin Orthod 2016;12(2):109-122.

13. Farronato G, Garagiola U, Carletti V, Cressoni P, Mortellaro C. Postsurgical orthodontic treatment planning: A case report with 20 years follow-up. J Oral Maxillofac Res 2011;2:4.

14. Kheckoyan D. Orthognathic surgery: General considerations Semin Plast Surg 2013;27:133-6.

15. Akan S, Kocadereli I, Tuncbilek G. Long-term stability of surgical-orthodontic treatment for skeletal class III malocclusion with mild asymmetry. J Oral Sci 2017;59:161-4.
16. Proffit WR, Phillips C, Dann C, Turvey TA. Stability after surgical-orthodontic correction of skeletal Class III malocclusion. I. Mandibular setback. Int J Adult Orthodon Orthognath Surg 1991;6:7-18.

17. Proffit WR, Turvey TA, Phillips C. Orthognathic surgery: A hierarchy of stability. Int J Adult Orthodon Orthognath Surg 1996;11:191-204.

18. Proffit WR, Turvey T, Philips C. The hierarchy of stability and predictability in orthognathic surgery with rigid fixation: an update and extension. Head Face Med 2007;3:21.

19. Radunovic V, Espeland L, Stenvik A. Retention: Type, duration and need for common guidelines. A survey of Norwegian orthodontists. Art Pract Dentofacial Enhanc 2013;14:110-7.

20. Pratt M, Kluemper G, Hartsfield J, Fardo D, Nash D. Evaluation of retention protocols among members of the American association of orthodontists in the United States. Am J Orthod Dentofacial Orthop 2011;140:520-6.

21. Renkema A, Sips E, Bronkhorst E, Jagtman A. A survey on orthodontic retention procedures in the Netherlands. Eur $J$ Orthod 2009;31:432-7.

22. Mai W, He J, Meng H, Jiang Y, Huang C, Li M, et al. Comparison of vacuum-formed and Hawley retainers: Asystematic review. Am J Ortho Dentofacial Orthop 2014;145:720-7.

23. Rossouw P. Retention maintenance-conclusion. Semin Orthod 2017;23:249-52 